\title{
Perviedade e complicações no seguimento de cateteres venosos totalmente implantáveis para quimioterapia
}

\author{
Patency and complications in the follow-up of totally implantable \\ catheters for chemotherapy \\ Robson Barbosa de Miranda ${ }^{1}$, Jocefábia Reika Alves Lopes ${ }^{2}$, Rafael Noronha Cavalcante ${ }^{2}$, \\ Ohannes Kafejian ${ }^{3}$
}

\section{Resumo}

Contexto: A disponibilidade de acesso venoso nos pacientes que recebem cursos prolongados de terapia citotóxica é de grande importância para o sucesso do tratamento. Os cateteres totalmente implantáveis vêm sendo cada vez mais utilizados para a referida terapia, proporcionando melhoria na qualidade de vida dos doentes.

Objetivo: Avaliar a perviedade e complicações dos cateteres venosos totalmente implantáveis instalados nos pacientes oncológicos.

Métodos: Estudo longitudinal retrospectivo com 74 pacientes submetidos a colocação de cateter totalmente implantável de janeiro de 2004 a fevereiro de 2007.

Resultados: Foram inseridos cateteres venosos totalmente implantáveis em 74 pacientes com idade média 48,9 anos, predominando o sexo feminino. As neoplasias mais prevalentes foram mama $(40,5 \%)$, cólon $(20,8 \%)$ e linfoma $(18,9 \%)$. Houve predomínio do acesso venoso pela via cervical $(74,3 \%)$, com utilização da veia jugular interna em $45,9 \%$ dos casos. Somente $13,5 \%$ dos acessos ocorreram por punção da veia subclávia. A duração média de uso dos cateteres foi de 335,33 dias. Trinta e seis doentes $(48,6 \%)$ mantiveram-se com o cateter após o término da quimioterapia. Sessenta e sete doentes $(90,5 \%)$ não apresentaram complicações. Entre as complicações precoces, houve um $(1,4 \%)$ pneumotórax e um $(1,4 \%)$ hematoma na loja de implantação. Entre as complicações tardias, ocorreram cinco $(6,7 \%)$ infecções. Foram retirados $10(13,5 \%)$ cateteres, cinco devido às complicações e cinco por término do tratamento. Houve $11(14,9 \%)$ óbitos de pacientes em decorrência do câncer, com o cateter funcionante.

Conclusão: Os resultados obtidos demonstram baixa taxa de complicações, confirmando a segurança e conveniência do uso dos acessos totalmente implantáveis em paciente em regime de quimioterapia.

Palavras-chave: Quimioterapia, cateter implantável, infecção, qualidade de vida.

\begin{abstract}
Background: Availability of venous access for patients that receive long-term cytotoxic therapy is of great importance to the success of treatment. Totally implantable devices have been increasingly more used for this therapy, providing improvement in the quality of life of patients.
\end{abstract}

Objective: To evaluate patency and complications of totally implantable catheters inserted in oncological patients.

Methods: Retrospective longitudinal study of 74 patients that underwent placement of totally implantable catheter from January 2004 throughout February 2007.

Results: Totally implantable catheters were placed in 74 patients with mean age of 48.9 years; the female gender was predominant. The most prevalent neoplasms were breast $(40.5 \%)$, colon $(20.8 \%)$ and lymphoma $(18.9 \%)$. Cervical access $(74.3 \%)$ was prevalent, using the internal jugular vein in $45.9 \%$ of cases. Only $13.5 \%$ of accesses were inserted via subclavian vein puncture. Mean duration of catheter use was 335.33 days. Thirty six ( $48.6 \%$ ) patients remained with the catheter after the chemotherapy was discontinued. There were no complications in $67(90.5 \%)$ patients. Among early complications, there was one $(1.4 \%)$ pneumothorax and one $(1.4 \%)$ hematoma. Among late complications, there were five $(6.7 \%)$ infections. Ten $(13.5 \%)$ catheters were removed, five due complications and five after ending the treatment. Eleven $(14.9 \%)$ patients died from cancer, and the catheters were still functioning.

Conclusion: The outcomes obtained show low rate of complications, confirming that use of totally implantable catheters is safe and effective for patients undergoing chemotherapy. of life.

1. Cirurgião vascular. Médico assistente, Disciplina de Angiologia e Cirurgia Vascular, Faculdade de Medicina, Fundação do ABC (FMABC), Santo André, SP.

2. Residentes de Cirurgia Vascular, Disciplina de Angiologia e Cirurgia Vascular, FMABC, Santo André, SP.

3. Professor titular, Disciplina de Angiologia e Cirurgia Vascular, FMABC, Santo André, SP.

Trabalho desenvolvido na Faculdade de Medicina da Fundação do ABC, São Paulo, Brasil, e apresentado no 37으 Congresso Brasileiro de Angiologia e Cirurgia Vascular.

Não foram declarados conflitos de interesse associados à publicação deste artigo.

Artigo submetido em 03.07.08, aceito em 31.10.08.

J Vasc Bras. 2008;7(4):316-320.

Copyright@ 2008 by Sociedade Brasileira de Angiologia e de Cirurgia Vascular 


\section{Introdução}

$\mathrm{O}$ acesso venoso central é recomendado no tratamento de pacientes submetidos a terapia endovenosa prolongada, notadamente em pacientes em uso de altas doses de quimioterapia, transplante de células sanguíneas, nutrição parenteral, entre outros ${ }^{1}$. Com o surgimento do cateter parcialmente implantável descrito por Broviac e modificado por Hickman durante 1970, o manejo dos pacientes oncológicos tornou-se mais fácil devido ao aumento da segurança deste em relação aos acessos periféricos ${ }^{2,3}$. Desde a introdução dos cateteres venosos totalmente implantáveis (CVTI) em 1980, vem ocorrendo um aumento no seu uso, revolucionando o tratamento e a qualidade de vida dos pacientes oncológicos $^{4,5}$.

O objetivo deste estudo é avaliar a perviedade e complicações dos CVTI instalados em pacientes submetidos a terapia citotóxica oncológica, no setor de quimioterapia, pela disciplina de Angiologia e Cirurgia Vascular da Faculdade de Medicina do ABC (FMABC).

\section{Métodos}

Foram estudados, retrospectivamente, 74 pacientes submetidos a colocação de CVTI, no período de janeiro de 2004 a fevereiro de 2007, no Hospital Estadual Mario Covas, da Faculdade de Medicina do ABC, São Paulo. Todos os cateteres foram colocados no centro cirúrgico sob anestesia local e com controle de locação usando fluoroscopia. Ao término do procedimento, testava-se fluxo e refluxo dos cateteres, seguido de heparinização do sistema com solução $50 \mathrm{UI} / \mathrm{mL}$ de heparina. Os pacientes faziam uso de antibioticoprofilaxia com $2 \mathrm{~g}$ de cefazolina antes da implantação do cateter.

As complicações foram classificadas em duas categorias: precoce (intra-operatório e pós-operatório antes do uso - primeiro mês) e tardia (complicações que ocorreram após o uso do cateter). Pacientes com infecção local de pele ou suspeita clínica de infecção sistêmica não eram submetidos a implante dos CVTI. Todos os cateteres com infecção foram retirados.

\section{Resultados}

A média de idade foi de 48,9 anos, variando de 18 a 74 anos, sendo 58 do sexo feminino $(78,4 \%)$ e 16 do sexo masculino $(21,6 \%)$. Desse grupo de pacientes, 13 apresentavam hipertensão arterial $(17,6 \%)$, cinco apresentavam trombose venosa profunda $(6,8 \%)$ e quatro eram diabéticos $(5,4 \%)$.

As neoplasias eram sólidas em $81,1 \%$ e hematológicas em $18,9 \%$. A neoplasia mamária foi a mais prevalente, seguida do câncer de cólon e linfoma (Tabela 1).

O acesso venoso por punção foi realizado em $63,5 \%$ dos casos. A dissecção venosa foi utilizada para acesso às veias jugulares externas e cefálicas. A via cervical (74,3\% dos casos) predominou, e a veia jugular interna $(45,9 \%)$ foi a mais utilizada. Somente $13,5 \%$ dos acessos ocorreram por punção subclávia, como segue na Tabela 2.

A duração média de uso dos cateteres foi de 335,33 dias, sendo que 36 pacientes $(48,6 \%)$ mantiveram-se com o cateter após o término da quimioterapia.

Não houve complicações em 67 pacientes $(90,5 \%)$. Houve duas complicações precoces, sendo um $(1,4 \%)$ pneumotórax e um $(1,4 \%)$ hematoma na loja de implantação. Entre as complicações tardias, observamos cinco infecções $(6,7 \%)$, conforme descrito na Tabela 3.

Foram retirados 10 cateteres $(13,5 \%)$, cinco devido às complicações $(6,7 \%)$ e cinco por término do tratamento $(6,7 \%)$.

No período estudado, 11 doentes (14,9\%) morreram em decorrência da doença neoplásica. Destes, todos estavam com seus cateteres pérvios e em funcionamento.

\section{Discussão}

Desde a sua introdução em 1980, os CVTI vêm sendo amplamente utilizados para aplicação de quimioterapia $^{4}$, especialmente nos pacientes com pobre circulação venosa periférica. Também têm sido utilizados em pacientes sob nutrição parenteral ${ }^{6-8}$ e hemodiálise ${ }^{9}$.

Os CVTI são constituídos por um cateter de silicone, cuja extremidade distal fica posicionada na junção da veia cava superior com o átrio direito, e a proximal conectada a um reservatório implantado no tecido celular subcutâneo. Isto permite uso seguro e repetido deste acesso vascular no tratamento endovenoso. Os CVTI oferecem vantagens em relação aos sistemas parcialmente implantáveis: baixas taxas de infecção e 
Tabela 1 - Tipos de neoplasias

\begin{tabular}{lc}
\hline Neoplasia & $\mathbf{n}(\%)$ \\
\hline Mama & $30(40,5)$ \\
Cólon & $15(20,8)$ \\
Linfoma & $14(18,9)$ \\
Reto & $4(5,4)$ \\
Outras & $11(14,9)$ \\
Total & $74(100)$ \\
\hline
\end{tabular}

Tabela 2 - Vias de acesso utilizadas para implante dos CVTI

\begin{tabular}{lccc}
\hline Vias de acesso & Dissecção & Punção & n (\%) \\
\hline Veia jugular interna direita & 0 & 21 & $21(28,3)$ \\
Veia jugular interna esquerda & 0 & 13 & $13(17,6)$ \\
Veia jugular externa direita & 9 & 0 & $9(12,2)$ \\
Veia jugular externa esquerda & 12 & 0 & $12(16,2)$ \\
Veia subclávia direita & 0 & 8 & $8(10,8)$ \\
Veia subclávia esquerda & 0 & 2 & $2(2,7)$ \\
Veia cefálica direita & 3 & 0 & $3(4,1)$ \\
Veia cefálica esquerda & 3 & 0 & $3(4,1)$ \\
Veia femoral direita & 0 & 3 & $3(4,1)$ \\
Total & $27(36,5)$ & $47(63,5)$ & $74(100)$ \\
\hline
\end{tabular}

Tabela 3 - Tipos de complicações

\begin{tabular}{lccc}
\hline Complicações & $\mathbf{n ~ ( \% )}$ & $\mathbf{l 1 . 0 0 0}$ dias de uso & Resolução \\
\hline Infecção & $5(6,7)$ & 0,19 & Antibiótico e remoção \\
Pneumotórax & $1(1,4)$ & 0,04 & Regressão espontânea \\
Hematoma & $1(1,4)$ & 0,04 & Tratamento clínico \\
Total & $7(9,4)$ & & \\
\hline
\end{tabular}

maior liberdade para as atividades físicas dos pacientes ${ }^{10-13}$.

Os CVTI estão associados a complicações como infecção, hematoma, pneumotórax, obstrução do cateter, trombose venosa profunda ${ }^{4,14-16}$, além de fratura com possível migração do cateter ${ }^{5}$.
Apesar das vantagens que os CVTI oferecem, esses dispositivos necessitam de manejo de profissionais experientes e podem ocasionalmente estar associados a complicações, como sangramento, pneumotórax e infecção, entre outras ${ }^{4}$. Dor local ou extravasamento subcutâneo durante a utilização do dispositivo alertam para a possibilidade de oclusão ou fratura do cateter. Um cateter 
Tabela 4 - Taxas de complicações após implantação de cateteres (comparação de nossos dados com os da literatura)

\begin{tabular}{lccc}
\hline Autor & N de cateteres $^{\text {o }}$ & Dias in situ & Infecção \\
\hline Chavát et al. $^{12}$ & 101 & 407 & $0,024 / 1.000$ \\
Wolosker et al. $^{4}$ & 519 & 353 & $0,23 / 1.000$ \\
Biffi et al. $^{1}$ & 376 & 473 & $0,16 / 1.000$ \\
Freytes et al. $^{6}$ & 134 & 305 & $0,02 / 1.000$ \\
Nossos dados & 74 & 335,33 & $0,19 / 1.000$ \\
\hline
\end{tabular}

fragmentado no coração pode permanecer assintomático por anos ${ }^{17}$.

As neoplasias predominantes foram as sólidas, não sendo encontrado nenhum caso de leucemia, visto ser uma patologia prevalente em pacientes jovens. Em nossa instituição, a cirurgia pediátrica é responsável pelo implante de CVTI em pacientes abaixo de 18 anos, conforme acordo entre as equipes cirúrgicas e a oncologia.

A via de acesso cervical (74,3\% dos casos) foi a mais utilizada, com predomínio da veia jugular interna $(45,9 \%)$ por punção, sendo observado que, dentro das sete complicações encontradas no nosso estudo, quatro ocorreram nos acessos realizados por dissecção.

As complicações observadas em nosso estudo foram similares às descritas nos trabalhos científicos publicados, conforme a Tabela 4.

Houve um caso de pneumotórax que foi acompanhado com exame físico e radiológico e tratado de forma conservadora com remissão espontânea do quadro no primeiro dia de pós-operatório. O baixo índice desta complicação provavelmente deve-se a pouca utilização, por punção, da via subclávia. O único caso de hematoma no local de punção foi tratado conservadoramente.

A bacteremia ou septicemia relacionada aos CVTI pode ocorrer por infecção no local da punção, contaminação da solução infundida ou mesmo por semeadura, por via hematogênica, a partir de um foco infeccioso à distância. A via de acesso femoral pode apresentar maior índice de infecção. Wolosker et al. ${ }^{18}$ observaram complicações infecciosas com freqüência de 0,69/1.000 dias de uso quando do implante de CVTI por via femoral. Abordagens mais conservadoras no tratamento desta complicação infecciosa têm sido propostas ${ }^{19}$. Nesta série, os cinco pacientes com suspeita clínica de infecção do cateter (hiperemia local, bacteremia, hipotensão arterial ou hemocultura positiva) foram submetidos a retirada do cateter e a instituição de terapia antibiótica.

Concluímos que as baixas taxas de complicação obtidas neste estudo, equivalentes à literatura, confirmam a segurança e conveniência do uso dos acessos totalmente implantáveis em pacientes em regime prolongado de quimioterapia.

\section{Referências}

1. Biffi R, Pozzi S, Agazzi A. Use of totally implantable central venous access ports for high-dose chemotherapy and peripheral blood stem cell transplantation: results of a monocentre series of 376 patients. Ann Oncol. 2004;15:296-300.

2. Broviac JW, Cole JJ, Scribner BH. A silicone rubber atrial catheter for prolonged parenteral alimentation. Surg Gynecol Obstet. 1973;136:602-6.

3. Hickman RO, Buckner CD, Clift RA, Sanders JE, Stewart P, Thomas ED. A modified right atrial catheter for access to the venous system in marrow transplant recipients. Surg Gynecol Obstet. 1979;148:871-5.

4. Wolosker N, Yazbek G, Nishinari K. Totally implantable venous catheters for chemotherapy: experience in 500 patients. São Paulo Med J. 2004;122:147-51.

5. Kapadia S, Parakh R, Grover T, Yadav A. Catheter fracture and cardiac migration of a totally implantable venous device. Indian J Cancer. 2005;42:155-7.

6. Di Carlo I, Cordio S, La Greca G. Totally implantable venous access devices implanted surgically: a retrospective study on early and late complications. Arch Surg. 2001;136:1050-3.

7. Kurul S, Saip P, Aydin T. Totally implantable venous-access ports: local problems and extravasation injury. Lancet Oncol. 2002;3:684-92.

8. Freytes CO. Indications and complications of intravenous devices for chemotherapy. Curr Opin Oncol. 2000;12:303-7.

9. Sandhu J. Dialysis ports: A new totally implantable option for hemodialysis access. Tech Vasc Interv Radiol. 2002;5:108-13.

10. Freytes CO, Reid P, Smith KL. Long-term experience with a totally implanted catheter system in cancer patients. J Surg Oncol. 1990;45:99-102. 
11. Gyves JW, Ensminger WD, Niederhuber JE, et al. A totally implanted injection port system for blood sampling and chemotherapy administration. JAMA. 1984;251:2538-41.

12. Carde P, Cosset-Delaigue MF, Laplanche A, Chareau I. Classical external indwelling central venous catheter versus totally implanted venous access systems for chemotherapy administration: a randomized trial in 100 patients with solid tumors. Eur J Cancer Clin Oncol. 1989;25:939-44.

13. Vanek VW. The ins and outs of venous access: Part I. Nutr Clin Pract. 2002;17:85-98.

14. Hackert T, Tjaden C, Kraft A, Sido B, Dienemann H, Buchler MW. Inatrapulmonal dislocation of a totally implantable venous access device. World J Surg Oncol. 2005;11:3-19.

15. Chang HM, Hsieh CB, Hsieh HF, et al. An alternative technique for totally implantable central venous access devices. A retrospective study of 1311 cases. Eur J Surg Oncol. 2006;32:90-3.

16. Charvát J, Linke Z, Horáèková M, Prausová J. Implantation of central venous ports with catheter insertion via the right internal jugular vein in oncology patients: single center experience. Support Care Cancer. 2006;14:1162-5.
17. Van Den Akker-Berman LM, Pinzur S, Aydinalp A, et al. Uneventful 25-year course of an intracardiac intravenous catheter fragment in the right heart. J Interv Cardiol. 2002;15:421-3.

18. Wolosker N, Yazbek G, Munia MA, Zerati AE, Langer M, Nishinari K. Totally implantable femoral vein catheters in cancer patients. Eur J Surg Oncol. 2004;30:771-5.

19. Koldehoff M, Zakrzewski JL. Taurolidine is effective in the treatment of central venous catheter-related bloodstream infections in cancer patients. Int $\mathbf{J}$ Antimicrob Agents. 2004;24:491-5.

Correspondência:

Robson Barbosa de Miranda

Rua Universal, 140/91

CEP 09060-050 - São Bernardo do Campo, SP

Tel.: (11) 4123.5677

E-mail: robsonmiranda@fluxo.com.br

\section{Mudança no endereço eletrônico da SBACV Nacional}

\section{Prezado Associado:}

O endereço do site da SBACV Nacional foi alterado para:

www.sbacv.com.br

O domínio dos e-mails também mudou:

@sbacv.com.br
Atualize hoje mesmo os endereços da SBACV Nacional no seu navegador e gerenciador de emails.

